\title{
Functional analysis of the gene expression profiles of colorectal cancer cell lines in relation to oxaliplatin and cisplatin cytotoxicity
}

\author{
DELPHINE MEYNARD, VALÉRIE LE MORVAN, JACQUES BONNET and JACQUES ROBERT \\ Laboratoire de Pharmacologie des Agents Anticancéreux, Institut Bergonié, CNRS FRE 2618, \\ Université Victor Segalen Bordeaux 2, 229 Cours de l'Argonne, 33076 Bordeaux-cedex, France
}

Received December 7, 2006; Accepted January 11, 2007

\begin{abstract}
The objective was to relate the gene expression profiles of colorectal cancer cells in culture to the in vitro cytotoxicity of cisplatin and oxaliplatin. We studied the gene expression profiles of six human colorectal cancer cell lines, using the Atlas Plastic Human 8K Microarray from Clontech, and related it to the in vitro cytotoxicities of oxaliplatin and cisplatin obtained by inhibition of exponential growth of cells. We calculated the Pearson's coefficients of correlation (r) between gene expression and drug $\mathrm{IC}_{50}$. A functional analysis was performed using the Gene Ontology Consortium database. Results were validated on a series of representative genes by real-time quantitative PCR. Validation of the significance of the coefficients of correlation was also performed using a leaveone-out analysis. We identified 394 genes whose expression was significantly correlated $(\mathrm{P}<0.05)$ to oxaliplatin cytotoxicity and 40 with cisplatin cytotoxicity. Three major functions were preferentially involved in oxaliplatin activity: protein synthesis, cell energetics and response to oxidative stress. No significant correlation was observed between oxaliplatin or cisplatin cytotoxicity and the expression of genes involved in DNA repair, cell proliferation or cell adhesion. A strongly significant correlation was found between the microarray and the rt-PCR approaches $\left(\mathrm{r}=0.968, \mathrm{P}<10^{-6}\right)$. The leave-one-out analysis showed that the same functions still appeared significantly involved in the activity of both drugs. Based on the functional analysis, we hypothesized that oxaliplatin would specifically form protein adducts during synthesis, thus exposing their thiol groups, which are known to be especially vulnerable to reactive oxygen species.
\end{abstract}

\section{Introduction}

The discovery of the antitumor properties of cisplatin (cisdiammino-dichloro-platinum II) (1) elicited major research

Correspondence to: Dr Jacques Robert, Laboratoire de Pharmacologie des Agents Anticancéreux, Institut Bergonié, CNRS FRE 2618, Université Victor Segalen, 229 cours de l'Argonne, 33076 Bordeaux-cedex, France

E-mail: robert@bergonie.org

Key words: colorectal cancer, in vitro cytotoxicity, gene expression profiling, platinum complexes programs aimed at the identification of other platinum derivatives with lower toxicity and higher activity, especially against cisplatin-resistant tumors (2). Among hundreds of compounds, the diaminocyclohexane $(\mathrm{DACH})$ derivatives were of special interest, and one of them, oxaliplatin (cis[oxalato(trans-1,2-cyclohexane diammine) platinum II]) was shown to be highly active against colorectal cancers, in combination with fluorouracil (3), whereas these tumors appeared primarily resistant to cisplatin (4). In contrast, germ cell tumors and squamous cell carcinomas, usually sensitive to cisplatin, did not appear to be as sensitive to oxaliplatin (3). Rixe et al (5) have established the in vitro cytotoxicity of oxaliplatin on the National Cancer Institute (NCI) panel of 60 human tumor cell lines. They observed that cisplatin and oxaliplatin had quite different cytotoxicity profiles. Interestingly, carboplatin, which is devoid of the DACH moiety, behaves similarly to cisplatin, while tetraplatin, which possesses a DACH moiety, has the same cytotoxicity profile as oxaliplatin.

Using the NCI databases on in vitro cytotoxicity profiles and basal gene expression profiles, we showed, in addition, that the determinants of the cytotoxicity of cisplatin and carboplatin on one hand, and of oxaliplatin and tetraplatin on the other hand, were clearly different (6). This infers that the mechanism of action of these two kinds of platinum derivatives may be different. Both compounds share common properties such as the formation of intrastrand and interstrand crosslinks following the preferential attack of G/C-rich DNA sites (7). However, despite the fact that both compounds are equally active, oxaliplatin induces less DNA lesions than cisplatin (8). This could be due to the fact that the presence of the DACH moiety renders oxaliplatin-induced DNA adducts more toxic than cisplatin-induced adducts; this could also be explained by a different cellular targeting of DACH-containing compounds.

Several studies have tentatively identified cellular markers of sensitivity to cisplatin, most often through the quantitative analysis of mRNA or protein populations. This has been performed on in vitro and in vivo models and only exceptionally on clinical samples. It has been shown, for instance, that the expression of genes involved in DNA repair (such as ERCC1, $X R C C 1$ or $X P D)$, apoptosis $(B C L 2, B A X)$ or drug detoxification (MRP1, metallothionein, GSTP1), was associated to sensitivity or resistance to cisplatin $(9,10)$. Genes associated with copper transport have also been shown to be involved in cisplatin sensitivity (11), and the inactivation of a splicing factor, SRPK1, has been shown to induce cisplatin resistance (12). 
Only a few studies have been devoted to oxaliplatin, most often in combination with fluorouracil. The expression of ERCC1 and $X P A$, two genes involved in nucleotide excision DNA repair, has been found to correlate with oxaliplatin resistance in vitro $(13,14)$. Samimi et al (15) recently compared the gene expression profiles of several cell lines selected for resistance to oxaliplatin to their wild-type sensitive counterparts and identified several genes and functions specifically associated with oxaliplatin resistance. Arango et al (16) performed a study aimed at correlating gene expression profiles in 30 colon tumor cell lines with the ability of oxaliplatin to induce apoptosis in these cell lines; however, no functional analysis has been performed on the sets of genes whose expression appeared to be predictive of oxaliplatin-induced apoptosis. In the clinical setting, it has only been shown that ERCCl tumor mRNA level was predictive for the survival of colorectal cancer patients treated with a combination of fluorouracil and oxaliplatin (17).

The identification of determinants of drug activity is an important challenge in oncology. In addition to oxaliplatin, several drugs are active against colorectal cancer, such as fluorouracil, irinotecan and raltitrexed, and this disease is no longer considered as globally chemoresistant as it was 15 years ago. The clinicians have now to choose, on empirical bases, a drug or a drug combination that should be administered to a given patient and there are currently no means to help the clinician make this choice in a rational way. It would be quite useful to be able to predict tumor response, at the individual level, to every potentially active drug, in order to maximize the probability of response while minimizing unwanted toxic effects. We were able, by mining the NCI databases, to identify some directions for future research on the determinants of oxaliplatin cytotoxicity (6); however, the NCI databases suffer from two major limitations: (i) drug cytotoxicity was evaluated with short-term growth inhibition tests, which represents only poorly the actual drug cytotoxicity (18); and (ii) gene expression profiles were established on a relatively low number of gene probes, a large number of which were not identified at that time. We found it necessary to extend this preliminary in silico study by an in vitro study, limited to the 6 colorectal cancer cell lines of the NCI panel, but with a re-evaluation of the cytotoxicity data by measuring inhibition of cloning efficiency, and with a panel of 8300 identified genes for gene expression profiling.

\section{Materials and methods}

Cell culture. The human colorectal tumor cell lines of the NCI panel, HCT15, HCT116, KM12, SW620, COLO205 and HT29 were purchased from the NCI Developmental Therapeutics Program tumor repository (Frederick, MD, USA). They were grown as monolayers in $75-\mathrm{cm}^{2}$ flasks (Nunc, Denmark) at $37^{\circ} \mathrm{C}$ in a humidified atmosphere containing $5 \% \mathrm{CO}_{2}$, using as a medium RPMI-1640 supplemented with $10 \%$ fetal calf serum and $1 \%$ antibiotic mixture, all purchased from Biochrom AG (Berlin, Germany). They were replicated every four days and the medium changed once in this time. Growth curves were first established by seeding 122,000 cells in $10-\mathrm{cm}^{2}$ Petri dishes (6-well multiplates, Nunc,), followed by cell counting every day using a Coulter Counter Z2 (Roissy, France).
Cytotoxicity tests. Cell growth inhibition was evaluated after seeding 4,000 cells in the wells of 96-well multiwell plates. Oxaliplatin (final concentrations, 0.05-5 $\mu \mathrm{M}$ ) or cisplatin (final concentrations, 0.5-20 $\mu \mathrm{M}$ ) was then added $24 \mathrm{~h}$ after seeding and the medium was changed against fresh medium $24 \mathrm{~h}$ later. After 48-h growth, cells were incubated for $4 \mathrm{~h}$ with 1-(4,5-dimethylthiazol-2-yl)-3,5-diphenylformazan (MTT, Sigma-Aldrich Chimie, Saint-Quentin-Fallavier, France) at a concentration of $0.5 \mathrm{mg} / \mathrm{ml}$. Formazan crystals were then dissolved in dimethylsulfoxide and the optical density measured at $550 \mathrm{~nm}$ using a multiplate photometer (Power Wave, BioTek Instruments). In these conditions, exponential cell growth was obtained throughout.

Cloning efficiency was evaluated after cell immobilization in NuSieve agar (Tebu, Le Perray en Yvelines, France). A $1 \%$ agar solution in complete culture medium was first poured into the wells of 6-well multiplates. After a gel was obtained, a $0.8 \%$ agar solution containing the cells in complete culture medium $(50,000$ per well) was poured on the first agar layer. After gelification, liquid culture medium was overlaid. In these conditions, the cells grew as discrete colonies containing 50-100 cells after 8 days. Oxaliplatin was added $24 \mathrm{~h}$ after seeding, at final concentrations ranging between 0.05 and $5 \mu \mathrm{M}$, and the medium was changed $24 \mathrm{~h}$ later and then every two days. On the 8th day, the colonies were colored with MTT, as described above. The plates were then scanned and the number of colonies was estimated using 1D image analysis software, version 3.6 (Kodak).

RNA extraction and gene expression profiling. Total RNAs were extracted from untreated cells in the exponential phase of growth, using TriReagent (Sigma-Aldrich). They were freed from any DNA contamination by DNase treatment and quantified by spectrophotometry at $260 \mathrm{~nm}$. Quality and integrity of RNAs was checked by agarose gel electrophoresis.

Synthesis of ${ }^{33} \mathrm{P}$-labeled cDNAs was performed with the Atlas Pure Total RNA Labeling System from Clontech - BD Biosciences (purchased from Ozyme, Saint-Quentin-enYvelines, France) according to the instructions of the manufacturer, using $10 \mu \mathrm{g}$ total RNA for each reverse transcription reaction. The quantity and the quality of the cDNA were evaluated by the measure of the radioactivity incorporated, in a liquid scintillation counter (Perkin Elmer, Courtaboeuf, France) and agarose gel electrophoresis. cDNAs were hybridized on BD Atlas Human 8K Microarrays (Clontech - BD Biosciences) according to the instructions of the manufacturer. The membranes were exposed to low energy screens (Amersham Biosciences, Orsay, France). The hybridization signals were recorded with a phosphoro-imager Typhoon 8610 (Molecular Imaging) and the intensity of each spot was quantified using Phoretix Array software (Nonlinear Dynamics).

Data analysis. The analysis of the results was basically performed using the Excel software (Microsoft). In a first step, the signal intensities originating from the duplicate spots were averaged, the background estimated and subtracted, and the results were normalized, first on the exogenous internal standards spotted on each membrane, then on the sum of the signals of the whole membrane. Background was set at the highest value of the distribution of the signals given by 
Table I. Primer sequences of the genes used for the rt-PCR validation of microarray data.

\begin{tabular}{|c|c|c|c|}
\hline Locus link \# & Gene name & 5' primer & 3' primer \\
\hline 405 & aryl hydrocarbon receptor nuclear translocator & CAGTGAAAAAGGAAGGTCAG & TTTACAGAGCCAAGTCCATT \\
\hline 506 & $\begin{array}{l}\text { ATP synthase, } \mathrm{H}^{+} \text {transporting, mitochondrial } \mathrm{F} 1 \text { complex, } \\
\text { ß polypeptide }\end{array}$ & CAATCAAAATTCCTGTTGGT & TACCAGTCACCAGAATTTCC \\
\hline 891 & cyclin B1 & TCTCCATTATTGATCGGTTC & CAGTCACAAAAGCAAAGTCA \\
\hline 977 & CD151 antigen & GTCTTTGGCATGATCTTCAC & GGTCTCAGCTCAGTAGTTGG \\
\hline 1351 & cytochrome c oxidase subunit VIII & TGTCACACCTGGAGACCTAC & GGAATTGAATGACATGATCC \\
\hline 1431 & citrate synthase & ACAGGTATCTTGGCTCTCAA & AGTACTTGGTTCGGCTGATA \\
\hline 1916 & eukaryotic translation elongation factor $1 \alpha 1$ & TCAGGTGATTATCCTGAACC & CGATCAATCTTTTCCTTCAG \\
\hline 2597 & glyceraldehyde-3-phosphate dehydrogenase & CCTGGTATGACAACGAATTT & GTGAGGGTCTCTCTCTTCCT \\
\hline 2810 & stratifin & CCAGTATTGAGCAGAAAAGC & CACCCTTCATCTTCAGGTAG \\
\hline 2936 & glutathione reductase & TACCCAAAAAGGTAATGTGG & TTCCTTAATAACACGCCAAT \\
\hline 3315 & heat shock $27 \mathrm{kD}$ protein 1 & CTTCACGCGGAAATACAC & ATGGTGATCTCGTTGGACT \\
\hline 3939 & lactate dehydrogenase A & AAACTCCAAGCTGGTCATTA & AAGCCACGTAGGTCAAGATA \\
\hline 4192 & midkine (neurite growth-promoting factor 2) & TCGCCAAAAAGAAAGATAAG & CAAACTCCTTCTTCCAGTTG \\
\hline 4609 & v-myc avian myelocytomatosis viral oncogene homolog & TCTGAGGAGGAACAAGAAGA & GTAGTTGTGCTGATGTGTGG \\
\hline 4697 & $\begin{array}{l}\text { NADH dehydrogenase (ubiquinone) } 1 \alpha \text { subcomplex, } 4 \\
(9 \mathrm{kD}, \mathrm{MLRQ})\end{array}$ & TTGTTGGGACAGAAATAACC & CCTGGTTAAGTGGAAAATTG \\
\hline 5315 & pyruvate kinase, muscle & AGCTTCCTTTCCTGTGTGTA & CTAACTCTTGCTGGCTGTTT \\
\hline 5695 & proteasome (prosome, macropain) subunit, $\beta$ type, 7 & GCAGATGCTTTTCAGGTATC & ATCCATGAGGATAGATGCTG \\
\hline 5962 & radixin & TGGTTCAGCTACTTTCCATT & TGCCCAGATTAATCCTTTTA \\
\hline 6161 & ribosomal protein $\mathrm{L} 32$ & ATCGTCAAAAAGAGAACCAA & GTTGCTTCCATAACCAATGT \\
\hline 6167 & ribosomal protein $\mathrm{L} 37$ & GTATAACTGGAGTGCCAAGG & TCTTGGGTTTAGGTGTTGTT \\
\hline 7386 & $\begin{array}{l}\text { ubiquinol-cytochrome c reductase, Rieske iron-sulfur } \\
\text { polypeptide } 1\end{array}$ & ATCCTGATAGGTGTTTGCAC & TCGTCACTGGTGAACTCATA \\
\hline 7447 & visinin-like 1 & GGATGAATTCAAAGAAGCTG & CCAAGCAATATTTGTGTGTG \\
\hline 7531 & $\begin{array}{l}\text { tyrosine } 3 \text {-monooxygenase/tryptophan } 5 \text {-monooxygenase } \\
\text { activation protein, } \varepsilon \text { polypeptide }\end{array}$ & AAATGGTGGAGTCAATGAAG & GTCTTCTCCTCCCTTGTTTT \\
\hline 8237 & ubiquitin specific protease 11 & ATCTATGTGTGGCTCTGTCC & AGATATCATCACGGTCCAAG \\
\hline 10313 & reticulon 3 & CGATCTGATTTTCTGGAGAG & GGATGAGGTAAGAAACCACA \\
\hline 10935 & peroxiredoxin 3 & TGCACCCTATTTTAAGGGTA & TGTAGGACACACAAAGGTGA \\
\hline
\end{tabular}

unexpressed genes. Only the genes whose expression was above background in at least one cell line were kept for further analysis (1325 genes). In a second step, the normalized signal intensities for each gene were compared in the six cell lines to the $\mathrm{IC}_{50} \mathrm{~s}$ of cisplatin and oxaliplatin, and the Pearson's coefficients of correlation were calculated and their significance evaluated based on 4 degrees of freedom. A functional analysis of the genes whose expression was significantly correlated to drug cytotoxicity was performed in a third step ( $r>0.73$ or $<-0.73, \mathrm{p}<0.05)$. This was done using the Gene Ontology Consortium annotations (http://www.godatabase.org/).

Quantitative real-time PCR. In order to validate the results obtained with microarrays, we have evaluated the expression of 26 different genes whose expression either did or did not correlate (positively or negatively) with drug cytotoxicity. It was necessary to set up a technique avoiding the use of reference genes such as GAPDH or $\beta$-actin, since the expression of these genes may also vary to a large extent and introduce biases. Our technique basically uses several independent retrotranscription reactions in order to minimize the variations known to occur during this reaction. A detailed description of the technique will be published separately and is only briefly described below.

Five independent retrotranscription reactions were performed with $2 \mu \mathrm{g}$ of total RNA from each cell line, the PowerScript ${ }^{\circledR}$ reverse transcriptase from Clontech, a dNTP mixture and N15 primers, both from Promega (Charbonnières, France). Each reaction was carried out in two separate tubes: the first one ( $5 \mu 1$ of the mix) in the presence of ${ }^{33} \mathrm{P}$-labeled dATP (Applied Biosystems, Courtaboeuf, France), the second one $(15 \mu 1$ of the mix $)$ in the presence of $10 \mathrm{mM}$ dATP. The yield of each reaction was estimated from ${ }^{33} \mathrm{P}$ incorporation and the same amount of cDNAs was sampled and pooled.

rt-PCR was conducted on equal amounts of cDNA for each cell line. It was performed with Taq polymerase Titanium ${ }^{\circledR}$ (Clontech) using primers specifically designed for generating a product of $<200 \mathrm{bp}$ and a $\mathrm{Tm}$ of $55^{\circ} \mathrm{C}$. Table I lists the selected genes with the primers used for the rt-PCR reactions. rt-PCR was carried out using a RotorGene ${ }^{\circledR}$ thermocycler from 
Corbett (purchased from Biotage, Uppsala, Sweden) and SYBR-Green ${ }^{\circledR}$ (Molecular Probes, Leiden, The Netherlands) as a fluorochrome. Absolute amounts of the cDNAs were determined from calibration curves generated by using known quantities of cDNA diluted four times successively by steps of $1 / 100$.

\section{Results}

Cytotoxicity evaluation. In order to evaluate the cytotoxicity of oxaliplatin and cisplatin against the six NCI colorectal cell lines, we performed growth inhibition assays using MTT as an endpoint to evaluate cell survival. The number of cells seeded in 96-well multiplates was low enough to ensure exponential growth during the whole process. For both drugs, the ratios between $\mathrm{IC}_{50}$ extreme values were 1:4 (Table II). The values obtained for cisplatin cytotoxicity strongly correlated with those retrieved from the NCI database $(\mathrm{r}=0.909, \mathrm{P}<0.01)$ but no significant correlation was observed for oxaliplatin $\mathrm{IC}_{50} \mathrm{~s}$.

We validated the $\mathrm{IC}_{50} \mathrm{~s}$ obtained using this method by comparing oxaliplatin data to those obtained using a clonogenic assay. In these conditions, oxaliplatin growth inhibition data appeared to correlate quite well with clonogenic data (Table II) but still considerably differed from those retrieved from the NCI database. We only performed growth inhibition tests to evaluate cisplatin cytotoxicity.

Identification of genes predictive for oxaliplatin and cisplatin cytotoxicity. Of the 8300 genes spotted in duplicate on the microarray, we selected those which gave a signal above background in at least five cell lines; this reduced the analysis to a total of 1325 gene probes. We then calculated the Pearson's coefficients of correlation (r) between the level of expression of the 1325 selected genes and the cytotoxicity of oxaliplatin and cisplatin, expressed as $-\log \left(\mathrm{IC}_{50}\right)$. We selected as significant $(\mathrm{P}<0.05)$ the $\mathrm{r}$ values $>0.73$ or $<-0.73$ and consequently identified 394 genes whose expression significantly correlated to oxaliplatin cytotoxicity (263 positively and 131 negatively) and 40 genes whose expression significantly correlated to cisplatin cytotoxicity ( 25 positively and 15 negatively).

Functional analysis. The functional analysis of the genes whose expression appeared to correlate to oxaliplatin or cisplatin cytotoxicity was performed using the Gene Ontology Consortium database, which attributes several functional identifiers to each gene. Of the 1325 genes giving at least one significant signal on one of the microarrays, 1296 had at least one function indicated in the Gene Ontology database. A total of 280 different functions could be assigned to the 394 genes significantly involved in oxaliplatin cytotoxicity, and 54 functions could be assigned to the 40 genes involved in cisplatin cytotoxicity. Sixty-three functions were mentioned at least 3 times for oxaliplatin (Table III), and 2 functions for cisplatin (DNA-dependent regulation of transcription, and metabolism).

We used a method based on principal component analysis to identify the functions significantly involved in oxaliplatin cytotoxicity, i.e. those for which the number of genes whose expression negatively or positively correlated to oxaliplatin
Table II. Growth inhibition patterns of cisplatin and oxaliplatin against the six colorectal cancer cell lines of the NCI panel.

\begin{tabular}{lccc}
\hline Cell line & $\begin{array}{c}\text { Cisplatin } \mathrm{IC}_{50} \\
(\mu \mathrm{M}) \\
\text { (growth inhibition tests) }\end{array}$ & $\begin{array}{c}\text { Oxaliplatin } \mathrm{IC}_{50} \\
(\mu \mathrm{M})\end{array}$ & $\begin{array}{c}\text { Oxaliplatin } \mathrm{IC}_{50} \\
(\mu \mathrm{M}) \\
\text { (cloning efficiency) }\end{array}$ \\
\hline COLO205 & $15.8 \pm 3.8$ & $0.66 \pm 0.34$ & 1.70 \\
HCT116 & 4.00 & $0.55 \pm 0.23$ & 0.62 \\
HCT15 & $7.45 \pm 0.64$ & $1.20 \pm 0.21$ & 1.90 \\
HT29 & $11.5 \pm 2.1$ & $0.37 \pm 0.05$ & 1.30 \\
KM12 & $13.3 \pm 3.5$ & $0.50 \pm 0.05$ & 0.98 \\
SW620 & $3.77 \pm 0.25$ & $0.30 \pm 0.08$ & 0.17 \\
\hline
\end{tabular}

$\mathrm{IC}_{50}$ significantly exceeded the expected random distribution. Three main groups of functions were thus identified: protein synthesis and degradation, including ribosomal proteins and translation initiation factors (55 genes involved over 102, $\mathrm{P}=2.6 \times 10^{-5}$ ), with positive correlations between gene expression levels and oxaliplatin cytotoxicity; cell energetics, including electron transport and glycolysis (27 genes involved over $46, \mathrm{P}=3.1 \times 10^{-3}$ ), with positive correlations between gene expression levels and oxaliplatin cytotoxicity; and response to oxidative stress (13 genes involved over $16, \mathrm{P}=7.2 \times 10^{-5}$ ), also with positive correlations between gene expression levels and oxaliplatin cytotoxicity. For all other functions, the number of genes whose expression significantly correlated to oxaliplatin cytotoxicity was not significantly different from that expected by random distribution, indicating no global involvement of this function in oxaliplatin activity. As a consequence, oxaliplatin cytotoxicity appeared to positively correlate with protein turnover, energy production, and antioxidant defense. No consistent relationship was found with any other cell function, such as DNA repair, cell proliferation or cell adhesion.

In contrast to oxaliplatin, a limited number of genes appeared to significantly correlate with cisplatin cytotoxicity and this did not allow a functional analysis of the functions involved. Consequently, we extended the number of genes studied from 40 to 200 , which corresponded to a $\mathrm{P}$ value of 0.11 . In these conditions, 22 functions were mentioned at least 3 times (Table IV) and 3 of them were significantly overrepresented in principal component analysis: these were ion transport $\left(\mathrm{P}=4.6 \times 10^{-3}\right)$, electron transport $\left(\mathrm{P}=3.7 \times 10^{-5}\right)$ and metabolism $\left(\mathrm{P}=2.6 \times 10^{-2}\right)$. It should be noted that the genes belonging to the electron transport function had an expression that negatively correlated with cisplatin cytotoxicity and positively correlated with oxaliplatin cytotoxicity.

Quantitative rt-PCR. In order to validate the gene expression levels estimated by microarray hybridization, we performed a series of 26 quantitative rt-PCR analyses on genes whose expression was shown to either correlate or not with oxaliplatin cytotoxicity. We then compared the Pearson coefficients of correlation between oxaliplatin $\mathrm{IC}_{50} \mathrm{~s}$ and the gene expression levels obtained with both techniques. These appeared to highly correlate ( $\mathrm{r}=0.968, \mathrm{P}<10^{-6}$, Fig. 1), justifying thus the use of 
Table III. Main functions involved in oxaliplatin cytotoxicity.

Function $\quad \begin{gathered}\text { No. of genes } \\ \text { involved }^{\mathrm{a}}\end{gathered}$ probes on the array
probsed

\begin{tabular}{l}
\hline Protein biosynthesis \\
Regulation of transcription, \\
DNA-dependent \\
Signal transduction \\
Development \\
Electron transport \\
Response to oxidative stress \\
Transport \\
Cell adhesion \\
Cell growth and/or \\
maintenance \\
Intracellular protein transport \\
Cell-cell signaling \\
G-protein coupled receptor \\
protein signaling pathway \\
Transcription from Pol II \\
promoter \\
Glycolysis \\
Cell proliferation \\
Immune response \\
Protein amino acid \\
phosphorylation \\
Regulation of cell cycle \\
Regulation of translational \\
initiation \\
Inflammatory response
\end{tabular}

Intracellular signaling cascade

Positive regulation of cell

proliferation

Protein folding

DNA replication

Metabolism

Neurogenesis

Transcription

Blood coagulation

Cellular defense response

Energy pathways

Proteolysis and peptidolysis

Regulation of transcription from Pol II promoter

Skeletal development

Small GTPase mediated

signal transduction

Synaptic transmission

Ubiquitin cycle

Ubiquitin-dependent protein catabolism

Carbohydrate metabolism

Cell motility
Table III. Continued.

\begin{tabular}{|c|c|}
\hline Function & $\begin{array}{c}\begin{array}{c}\text { No. of genes } \\
\text { No. of expressed } \\
\text { involved }^{\mathrm{a}}\end{array} \text { probes on the array }\end{array}$ \\
\hline
\end{tabular}

\begin{tabular}{lll}
\hline Cell surface receptor linked & 4
\end{tabular}

signal transduction

Chemotaxis

Cytokinesis

Defense response

Ion transport

Negative regulation of cell

proliferation

Neuropeptide signaling pathway

Positive regulation of

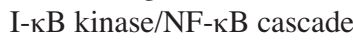

Protein complex assembly

Response to pest/pathogen/

parasite

Response to virus

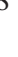

RNA splicing

$\begin{array}{rr}4 & 8 \\ 4 & 10 \\ 4 & 14 \\ 4 & 19 \\ 4 & 20\end{array}$

Wnt receptor signaling pathway

Apoptosis

Cell cycle arrest

Central nervous system

development

DNA repair

Humoral immune response

Mitosis

Morphogenesis

Negative regulation of transcription from Pol II promoter

Nuclear mRNA splicing, via spliceosome

RNA processing

Visual perception

$4 \quad 8$

49

$4 \quad 15$

$4-5$

$4 \quad 5$

$4 \quad 13$

$4 \quad 7$

$3 \quad 17$

$3 \quad 7$

39

$3 \quad 10$

35

36

$3 \quad 12$

39

$3 \quad 11$

36

$3 \quad 8$

The functions are those indicated in the Gene Ontology Consortium database. The same gene may be attributed several different functions. Only the functions mentioned at least three times are indicated in this table. ${ }^{a}$ No. of genes whose expression significantly correlated with oxaliplatin cytotoxicity and having the function indicated (total no. of genes: 394 ). ${ }^{\text {b No. }}$ of genes whose expression was detected on the array and having the function indicated (total no. of genes: 1296). *Significant overrepresentation of the function among the genes correlated with drug cytotoxicity.

the Clontech microarrays for evaluating the simultaneous expression of several thousands of genes. Only three genes have been misclassified by the microarray technique, with $\mathrm{r}$ values $>0.73$, whereas $r$ was $<0.73$ (but $>0.55$ ) with the rtPCR technique.

'Leave-one-out' or 'Jacknife' analysis. In order to evaluate the actual significance of the coefficients of correlation obtained, we performed a 'leave-one-out' analysis as follows: of the set of 6 cell lines, one was withdrawn after the other and the 
Table IV. Main functions involved in cisplatin cytotoxicity.

\begin{tabular}{|c|c|}
\hline Function & $\begin{array}{c}\begin{array}{c}\text { No. of genes } \\
\text { involved }^{\mathrm{a}}\end{array} \\
\text { present on array }\end{array}$ \\
\hline
\end{tabular}

\begin{tabular}{|c|c|c|c|}
\hline $\begin{array}{l}\text { Regulation of transcription, } \\
\text { DNA-dependent }\end{array}$ & 19 & 103 & \\
\hline Signal transduction & 10 & 82 & \\
\hline Electron transport & 12 & 25 & $*$ \\
\hline $\begin{array}{l}\text { Protein amino acid } \\
\text { phosphorylation }\end{array}$ & 9 & 2 & \\
\hline Ion transport & 9 & 18 & $*$ \\
\hline Transport & 8 & 43 & \\
\hline Metabolism & 8 & 21 & $*$ \\
\hline Cell proliferation & 7 & 27 & \\
\hline Protein folding & 5 & 19 & \\
\hline Cell growth and/or maintenance & 5 & 29 & \\
\hline Synaptic transmission & 4 & 20 & \\
\hline Sodium ion transport & 4 & 9 & \\
\hline $\begin{array}{l}\text { Regulation of transcription } \\
\text { from Pol II promoter }\end{array}$ & 4 & 21 & \\
\hline Immune response & 4 & 34 & \\
\hline $\begin{array}{l}\text { G-protein coupled receptor } \\
\text { protein signaling pathway }\end{array}$ & 4 & 33 & \\
\hline Cell adhesion & 4 & 35 & \\
\hline Carbohydrate metabolism & 4 & 14 & \\
\hline Transcription & 3 & 15 & \\
\hline $\begin{array}{l}\text { Positive regulation of cell } \\
\text { proliferation }\end{array}$ & 3 & 19 & \\
\hline $\begin{array}{l}\text { Negative regulation of cell } \\
\text { proliferation }\end{array}$ & 3 & 19 & \\
\hline Intracellular protein transport & 3 & 20 & \\
\hline Cell cycle & 3 & 12 & \\
\hline
\end{tabular}

The functions are those indicated in the Gene Ontology Consortium database. The same gene may be attributed several different functions. Only the functions mentioned at least three times are indicated in this table. ${ }^{a}$ No. of genes whose expression significantly correlated with oxaliplatin cytotoxicity and having the function indicated (total no. of genes: 394$).{ }^{b}$ No. of genes whose expression was detected on the array and having the function indicated (total no. of genes: 1296). "Significant overrepresentation of the function among the genes correlated with drug cytotoxicity. The total numbers of genes whose expression significantly correlated with cisplatin cytotoxicity was 40 but we extended the functional study to the first 200 genes, whose expression less significantly correlated to cisplatin cytotoxicity.

coefficients of correlation recalculated with the remaining set of 5 cell lines. In these conditions, there remained 105 genes whose expression still significantly correlated with the cytotoxicity of oxaliplatin $(r>0.80$ or $<-0.80, \mathrm{P}<0.05)$, 81 positively and 24 negatively. A functional analysis was performed as for the first set of data and gave the same results.

In addition, the 10 genes whose expression correlated with drug cytotoxicity with the highest coefficients of correlation were retained after each test. Five genes for oxaliplatin and six genes for cisplatin were eventually identified as belonging

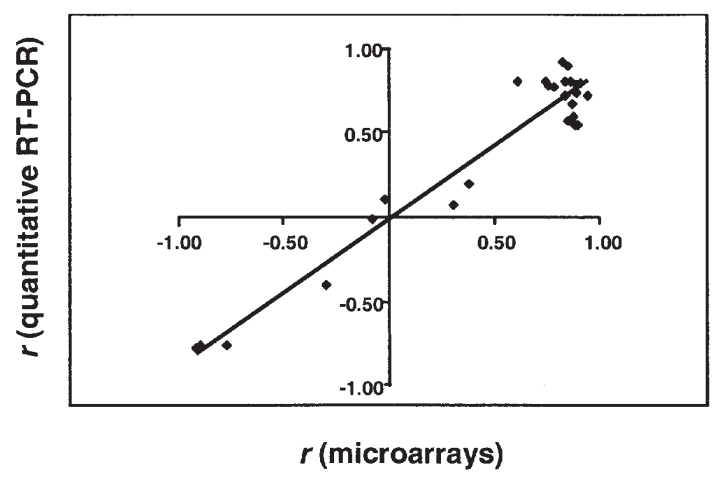

Figure 1. Comparison of the Pearson coefficients of correlation between oxaliplatin cytotoxicity and gene expression data as obtained either from Clontech microarray data (abscissa) or from quantitative real-time PCR (ordinates). $\mathrm{N}=26, \mathrm{r}=0.968, \mathrm{P}<10^{-6}$.

Table V. Genes whose expression is the most significantly related to oxaliplatin cytotoxicity.

\begin{tabular}{clc}
\hline Locus link \# & \multicolumn{1}{c}{ Gene name } & $\mathrm{r}$ \\
\hline 1667 & defensin, $\alpha$ 1, myeloid-related sequence & -0.99 \\
22977 & $\begin{array}{l}\text { aldo-keto reductase family 7, member A3 } \\
\text { (aflatoxin aldehyde reductase) }\end{array}$ & -0.98 \\
588 & B-cell growth factor 1 (12 kD) & -0.98 \\
1537 & cytochrome c-1 & 0.96 \\
8301 & $\begin{array}{l}\text { Clathrin assembly lymphoid-myeloid } \\
\text { leukemia gene }\end{array}$ & 0.97 \\
\hline
\end{tabular}

Table VI. Genes whose expression is the most significantly related to cisplatin cytotoxicity.

\begin{tabular}{|c|c|c|}
\hline Locus link \# & Gene name & $\mathrm{r}$ \\
\hline 432 & asialoglycoprotein receptor 1 & 0.97 \\
\hline 412 & $\begin{array}{l}\text { steroid sulfatase (microsomal), arylsulfatase C, } \\
\text { isozyme S }\end{array}$ & 0.93 \\
\hline 7262 & $\begin{array}{l}\text { tumor suppressing subtransferable } \\
\text { candidate } 3\end{array}$ & 0.96 \\
\hline 5959 & retinol dehydrogenase 5 (11-cis and 9-cis) & 0.93 \\
\hline 27091 & $\begin{array}{l}\text { calcium channel, voltage-dependent, } \\
\gamma \text { subunit } 5\end{array}$ & 0.81 \\
\hline 5711 & $\begin{array}{l}\text { proteasome (prosome, macropain) } \\
26 \mathrm{~S} \text { subunit, non-ATPase, } 5\end{array}$ & -0.82 \\
\hline
\end{tabular}

to at least two different leave-one-out tests (Tables V and VI). These genes were considered as the most predictive of drug cytotoxicity. An equation relating their expression to oxaliplatin $\mathrm{IC}_{50}$ in the six cell lines was built; the coefficient of correlation between actual and predicted $\mathrm{IC}_{50} \mathrm{~s}$ for each cell line was 0.987 for oxaliplatin $\left(\mathrm{P}<10^{-4}\right)$ and 0.931 for cisplatin $\left(\mathrm{P}<2 \times 10^{-3}\right)$ (Fig. 2). 

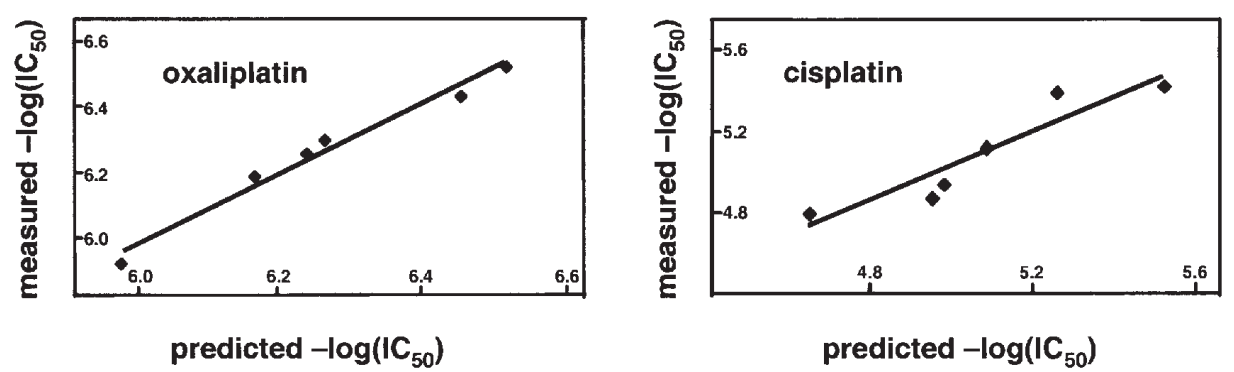

Figure 2. Comparison between the $\mathrm{IC}_{50} \mathrm{~s}$ of oxaliplatin and cisplatin vis-à-vis the six cell lines (expressed as the opposite of the logarithm of their value expressed in mol/l) obtained experimentally (ordinates) and from the expression levels of the 5 or 6 genes that most significantly correlated with cytotoxicity (abscissas). For oxaliplatin, $\mathrm{r}=0.987, \mathrm{P}<10^{-4}$; for cisplatin, $\mathrm{r}=0.931, \mathrm{P}<2 \times 10^{-3}$.

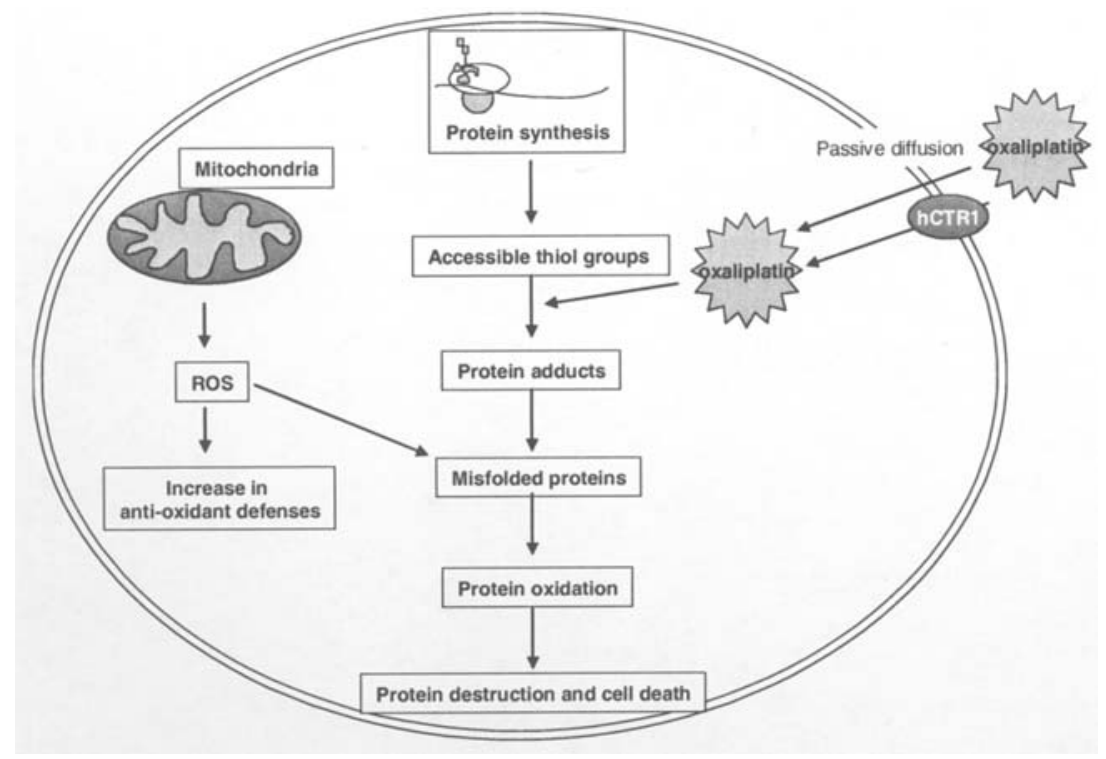

Figure 3. Hypothesis of the mechanism involved in oxaliplatin cytotoxicity. After entry in the cell, oxaliplatin would especially target the thiol groups of nascent proteins, which would be thus particularly sensitive to the reactive oxygen species (ROS) produced by mitochondrial respiration. The resulting protein oxidation would be at the origin of cell death.

\section{Discussion}

The first-line treatment of advanced colorectal cancers by chemotherapy induces at best $30-50 \%$ responses when it combines several active drugs; moreover, this is often at the expense of limited tolerance. It would be, therefore, of uppermost interest to identify predictive molecular markers allowing the optimization of this treatment by a rational selection of the drugs prescribed. We wanted, in this study, to focus our investigations on oxaliplatin, one of the major active drugs of advanced colorectal cancers, and to compare it with cisplatin, which is devoid of activity in this malignancy. We used an in vitro model represented by the 6 colorectal carcinoma cell lines of the NCI, and we first re-estimated the cytotoxicity of both drugs. The $\mathrm{IC}_{50}$ values we obtained for cisplatin were comparable to those obtained by the NCI; however, those obtained for oxaliplatin were quite different from those retrieved from the NCI database, and the two sets of values did not even significantly correlate. We hypothesized that this was due to the fact that the $\mathrm{IC}_{50}$ values determined by the NCI were based on a sulforhodamine assay, which evaluates the protein amount in the cell culture. We have shown that protein synthesis rate is a strong determinant of oxaliplatin cytotoxicity; in addition, oxaliplatin induces an alteration in protein synthesis rate in some cell lines (data not shown). As a conclusion, a growth inhibition test based on protein estimation might not be reliable for the estimation of oxaliplatin cytotoxicity. The significant correlation between $\mathrm{IC}_{50} \mathrm{~s}$ evaluated by MTT-based growth inhibition and by clonogenic survival assay argues in favor of this interpretation.

Using the Clontech Atlas Human 8K microarray, we identified 394 genes whose expression significantly correlated with oxaliplatin cytotoxicity and 40 whose expression significantly correlated with cisplatin cytotoxicity. There are currently a large number of facilities available for establishing gene expression profiles, but very few cross-comparisons have been performed to evaluate their respective performance. We validated the use of the Clontech microarrays by selecting 26 genes whose expression did or did not correlate with the cytotoxicity of oxaliplatin and comparative estimation of the Pearson coefficients of correlation with data originating from both techniques. This comparison is, therefore, a functional 
one, not taking into account the relative levels of expression, which are impossible to compare when using different controls and references, but relying on the links between the expression of a gene in 6 cell lines and a functional parameter (oxaliplatin $\left.\mathrm{IC}_{50}\right)$. We obtained a highly significant correlation showing the reliability of the cDNA arrays used in this study.

Based on the analysis of gene expression profiles and the computation of Pearson coefficients of correlation between drug cytotoxicity and gene expression profiles, and using principal component analysis, we were able to identify the gene functions, as defined by the Gene Ontology Consortium, which were significantly overrepresented. Several functions appeared to be involved in cell sensitivity to oxaliplatin and not in cell sensitivity to cisplatin. There was a relationship between oxaliplatin cytotoxic activity and: (i) protein synthesis, (ii) energy production by both glycolysis and mitochondrial electron transport, and (iii) the presence of oxidative stress. The importance of protein synthesis in oxaliplatin's action could be related to the fact that DACH-containing platinum compounds form much more protein adducts than other platinum derivatives (19). The addition events would mainly occur at the polypeptide translation step, during which the thiol groups are particularly accessible. Cells having an intense protein synthesis activity would be, as a consequence, especially exposed to the formation of such adducts, which would in turn hinder normal protein folding. Misfolded proteins are especially sensitive to oxidation because of a better accessibility to reactive oxygen species (ROS). Such ROS are especially formed during electron transfer reactions which occur as a side effect of mitochondrial respiration (20). They are toxic to the cells and are detoxified by the anti-oxidant defenses; the balance between production and elimination of ROS constitutes the basal oxidative stress (20). As a consequence, the activity of the mitochondrial respiratory chain would correlate with an increase in the oxidation of the proteins damaged by oxaliplatin: this would explain why energy production appears to positively correlate with oxaliplatin cytotoxicity, and why the cellular anti-oxidant defenses, which are solicited by the formation of reactive oxygen species, also positively correlate with oxaliplatin cytotoxicity.

Protein synthesis did not appear to be involved in the cytotoxicity of cisplatin, which corroborates the observations about the fundamental differences between the clinical properties of classical and $\mathrm{DACH}$-containing platinum derivatives that we have already emphasized (6). Electron transport appeared to be related to cisplatin cytotoxicity, but the significant correlations observed between gene expression and cytotoxicity were always negative, whereas there was significantly positive correlations between oxaliplatin cytotoxicity and the expression of genes involved in electron transport.

Among the genes that have been shown to play a role in sensitivity or resistance to platinum compounds by other studies (9-15), we found only a few that correlated with the cytotoxicity of cisplatin or oxaliplatin in our study: the copper transporter ATP7B, the catalytic subunit of DNA polymerase $\zeta$ REV3L and the nucleotide excision repair ERCC4 protein negatively correlated with cisplatin cytotoxicity $(\mathrm{r}=-0.72,-0.89$ and -0.79 respectively), whereas COX17 (copper chaperone) and SRPK1 (a splicing factor) positively correlated with oxaliplatin cytotoxicity ( $\mathrm{r}=0.78$ and 0.80 , respectively). Unexpectedly, the detoxification proteins ABCC1 (multidrug resistance protein 1) and GSTP1 (glutathione S-transferase $\pi$ ) positively correlated with the cytotoxicity of oxaliplatin ( $\mathrm{r}=0.81$ for both), emphasizing again the unusual behavior of this anticancer drug. Our results show that no DNA repair gene plays a predictive role in cisplatin or oxaliplatin activity. This does not mean that adduct formation and DNA damage are not responsible for the cytotoxicity of platinum compounds, but rather that the basal level of expression of the genes involved in DNA repair is high enough to insure proper repair in unselected cells. DNA repair proteins were not associated to oxaliplatin resistance in the study of Samimi et al (15).

Arango et al (15) performed a study also intended to identify predictors of oxaliplatin response by analysis of gene expression profiles. However, the cytotoxicity endpoint they used was apoptosis induction, which represents only the terminal aspect of drug action. It is not surprising, therefore, that only one gene appeared in common in the sets of 254 and 394 predictive genes respectively identified in their study and ours. In addition, no functional analysis was performed, and a short survey of their results shows that there was no global involvement of either the gene functions classically involved in platinum activity (DNA repair), or those we identified as predictors of oxaliplatin cytotoxicity (e.g. protein synthesis).

With a set of 6 cell lines, it was not possible to develop a predictability model based upon the successive establishment of a training set and a validation set. In order to analyze the performance of the genes identified, we rather performed a 'leave-one-out' test in order to eliminate the correlations that appear to be only significant when driven by a single cell line. Most of the genes identified as non-cell line-dependent correspond to the functions significantly involved in oxaliplatin cytotoxicity as determined using principal component analysis, confirming thus the importance of these functions in drug activity. This allowed the identification of genes whose expression appeared to most significantly correlate with drug cytotoxicity. In addition, the 5- and 6-gene sets obtained for oxaliplatin and cisplatin, respectively, appear to be highly predictive of drug activity. It is difficult to relate these genes to what is known concerning the mechanism of action of platinum analogues or to the mechanisms of resistance against these drugs; it appears, however, that those that negatively correlated with oxaliplatin cytotoxicity are involved in defense response to aggression. Special attention should be brought to these genes when searching for clinical correlations between tumor gene expression profiles and patients' responses to oxaliplatin or cisplatin.

In conclusion, our study allowed the identification, not only of genes, but of functions involved in cell response to oxaliplatin. The main conclusion is that the mechanism of action of oxaliplatin is distinct from that of cisplatin and is likely to involve the formation of protein adducts, especially on nascent proteins. Fig. 3 shows the present state of this hypothesis. We are currently studying the effect of oxaliplatin and cisplatin on gene expression profiles to confirm these hypotheses. 


\section{Acknowledgements}

This study was supported by grants from the Ligue Nationale contre le Cancer, comités de la Charente et de la CharenteMaritime and by Debiopharm S.A. DM was supported by a fellowship from the Association pour la Recherche sur le Cancer.

\section{References}

1. Rosenberg B: Fundamental studies with cisplatin. Cancer 55: 2303-2316, 1985

2. Hamilton TC, O'Dwyer PJ and Ozols RF: Platinum analogues in preclinical and clinical development. Curr Opin Oncol 5: 1010-1016, 1993

3. Misset JL, Bleiberg H, Sutherland W, Bekradda M and Cvitkovic E: Oxaliplatin clinical activity: a review. Crit Rev Oncol Hematol 35: 75-93, 2000.

4. Kemeny N, Israel K, Niedzwiecki D, et al: Randomized study of continuous infusion fluorouracil versus fluorouracil plus cisplatin in patients with metastatic colorectal cancer. J Clin Oncol 8: 313-318, 1990.

5. Rixe O, Ortuzar W, Alvarez M, Parker R, Reed E, Paull K and Fojo T: Oxaliplatin, tetraplatin, cisplatin and carboplatin: spectrum of activity in drug-resistant cell lines and in the cell lines of the National Cancer Institute anticancer drug screen panel. Biochem Pharmacol 52: 1855-1865, 1990.

6. Vekris A, Meynard D, Haaz MC, Bayssas M, Bonnet J and Robert J: Determinants of the clinical activity of platinum compounds: the contribution of in silico research. Cancer Res 64: 356-362, 2004.

7. Di Francesco AM, Ruggiero A and Riccardi R: Cellular and molecular aspects of drugs of the future: oxaliplatin. Cell Mol Life Sci 59: 1914-1927, 2002.

8. Raymond E, Faivre S, Woynarowski JM and Chaney SG: Oxaliplatin: mechanism of action and antineoplastic activity. Semin Oncol 25 (suppl 5): 4-12, 1998.
9. Perez RP: Cellular and molecular determinants of cisplatin resistance. Eur J Cancer 34: 1534-1542, 1998

10. Niedner H, Christen R, Lin X, Kondo A and Howell SB: Identification of genes that mediate sensitivity to cisplatin. Mol Pharmacol 60: 1153-1160, 2001.

11. Komatsu M, Sumizawa T, Mutoh M, et al: Copper-transporting P-type adenosine triphosphatase (ATP7B) is associated with cisplatin resistance. Cancer Res 60: 1312-1316, 2000.

12. Schenk PW, Boersma AW, Brandsma JA, et al: SKY1 is involved in cisplatin-induced cell kill in Saccharomyces cerevisiae, and inactivation of its human homologue, SRPK1, induces cisplatin resistance in a human ovarian carcinoma cell line. Cancer Res 61: 6982-6986, 2001.

13. Hector S, Bolanowska-Higdon W, Zdanowicz J, Hitt S and Pendyala L: In vitro studies on the mechanisms of oxaliplatin resistance. Cancer Chemother Pharmacol 48: 398-406, 2001.

14. Arnould S, Hennebelle I, Canal P, Bugat R and Guichard S: Cellular determinants of oxaliplatin sensitivity in colon cancer cell lines. Eur J Cancer 39: 112-119, 2003.

15. Samimi G, Manorek G, Castel R, et al: cDNA microarray-based identification of genes and pathways associated with oxaliplatin resistance. Cancer Chemother Pharmacol 55: 1-11, 2005.

16. Arango D, Wilson AJ, Shi Q, et al: Molecular mechanisms of action and prediction of response to oxaliplatin in colorectal cancer cells. Br J Cancer 91: 1931-1946, 2004.

17. Shirota Y, Stoehlmacher J, Brabender J, et al: ERCC1 and thymidylate synthase mRNA levels predict survival for colorectal cancer patients receiving combination oxaliplatin and fluorouracil chemotherapy. J Clin Oncol 19: 4298-4304, 2001.

18. Brown JM and Wouters BG: Apoptosis, p53, and tumor cell sensitivity to anticancer agents. Cancer Res 59: 1391-1399, 1999.

19. Chaney S: The chemistry and biology of platinum complexes with the 1,2-diaminocyclohexane carrier ligand. Int J Oncol 6: 1291-1305, 1995

20. Dukan S, Farewell A, Ballesteros M, Taddei F, Radman M and Nystrom T: Protein oxidation in response to increased transcriptional or translational errors. Proc Natl Acad Sci USA 97: 5746-5749, 2000 . 